Nephron 1977;19:185-199

\title{
The Treatment of Lupus Nephropathy
}

We are introducing a new series - Unsolved Problems in Nephrology - and have elected to examine current opinion on the treatment of renal lupus. Ten years ago the subject was thought to be fairly well defined, but as our knowledge of the renal manifestations of the disease has progressed, coupled with advances in immunology and electron microscopy, it is apparent that we know a good deal less about the indicated therapy in this disease in its various renal forms. We have therefore invited four groups of internationally recognised authorities in the field to set down their views in the form of editorial articles. The reader will benefit from an up-to-date statement of the art as it is seen by the experts at present.

G.M.Berlyne S. Giovannetti 